\title{
Nanoparticle Chains for Plasmonic Band Engineering
}

Johannes Schultz ${ }^{1}$, Daniel Schletz ${ }^{2}$, Pavel Potapov ${ }^{3}$, Anja Maria Steiner ${ }^{2}$, Jonas Krehl ${ }^{1}$, Tobias König ${ }^{2}$, Martin Mayer ${ }^{2}$, Axel Lubk ${ }^{4}$ and Andreas Fery ${ }^{2}$

${ }^{1}$ IFW-Dresden, United States, ${ }^{2}$ IPF-Dresden, United States, ${ }^{3}$ IFW-Dresden, Dresden, Sachsen, Germany, ${ }^{4}$ IFW Dresden, Germany, United States

Localized Surface Plasmons (LSPs) are collective charge oscillations on the surface of nanoparticles (NPs). They can be excited at interfaces between media with opposite sign of the corresponding dielectric functions; and hence are very sensitive to the materials dielectric function $\varepsilon$ and the particle geometry. The induced plasmonic fields are strongly enhanced and confined to the interface, which makes LSPs attractive for several applications.

Of special interest are ordered arrangements of NPs forming one-, two- or three-dimensional photonic crystals. Depending on the symmetry and coupling strength they can support bands of hybridized LSPs. In order to tune these delocalized plasmon bands for certain applications (such as wave guides) precise control of the coupling is needed. This can be achieved in principle by tuning the interparticle gap, the NP shape or material. Here, particular complex band structures including band gaps and crossing bands may be generated by varying the coupling strength periodically. Ubiquitous disorder in such systems, on the other hand, leads to spectral and spatial localization of the LSP modes similar to localization phenomena in electronic systems.

To explore the above-mentioned delocalization and localization effects, we studied one-dimensional homogeneous and heterogeneous chains of coupled nanospheres with the diameter $~ 70 \mathrm{~nm}$ by Scanning Transmission Electron Microscopy (STEM) in combination with Electron Energy Loss Spectroscopy (EELS) [1]. The chains are self-assembled using a wrinkled template. In order to describe the plasmonic response of the periodic arrangements theoretically we use Boundary Element Method (BEM) simulations as well as a Discrete-Dipole-Approximation (DDA) model.

Fig. 1 shows the appearance of several hybridized LSP modes with increasing number of nanospheres in a homogeneous chain of Au NPs. Starting from short chains sustaining only a few strongly localized LSP modes, the difference in excitation energies of the modes decreases with increasing number of NPs. At a chain length of about 10 NPs, the plasmon energies start to overlap and the LSP modes tends to delocalize spectrally and spatially. For a quasi-infinite chain, this behavior results in a completely delocalized quasicontinuous plasmon band [2]. We characterize this limit and calculate pertaining excitation energies, which optimize the signal transport along the chains.

In a second step, we assemble statistical combinations of monodisperse gold and silver nanospheres to heterogeneous chains of different lengths [3]. Fig. 2 displays a subset of heterogeneous four NP chains with different Ag-Au percentage. Starting from the pure silver and gold chain respectively one can observe the hybridized modes indicated by circles, triangles, and spheres in the heterogeneous chains at an intermediate spectral position with respect to the pure chains. Applying an angular momentum classification known from Mie theory these modes correspond to strong coupling $\mathrm{p}_{x}$ modes $(l=1, x$ direction parallel to the chain respectively). On the other hand, one can observe non-hybridized modes like $\mathrm{p}_{y}, \mathrm{p}_{z}$ and higher order modes $(l>1)$ indicated by diamonds (pentagons) in case of gold (silver). 
All in all, our results confirm the emergence of a large variety of hybridized surface plasmons in heterogeneous NP arrangements. Further exploitation in applications hinges on better control of the NP arrangement and reduction of the ubiquitous loss.

This project was funded by the European Research Council (ERC) under the Horizon 2020 research and innovation program of the European Union (grant agreement no. 715620). Furthermore is was funded by the Deutsche Forschungsgemeinschaft (DFG, German Research Foundation) under Germany's Excellence Strategy through Würzburg-Dresden Cluster of Excellence on Complexity and Topology in Quantum Matter - ct.qmat (EXC 2147, project-id 390858490).

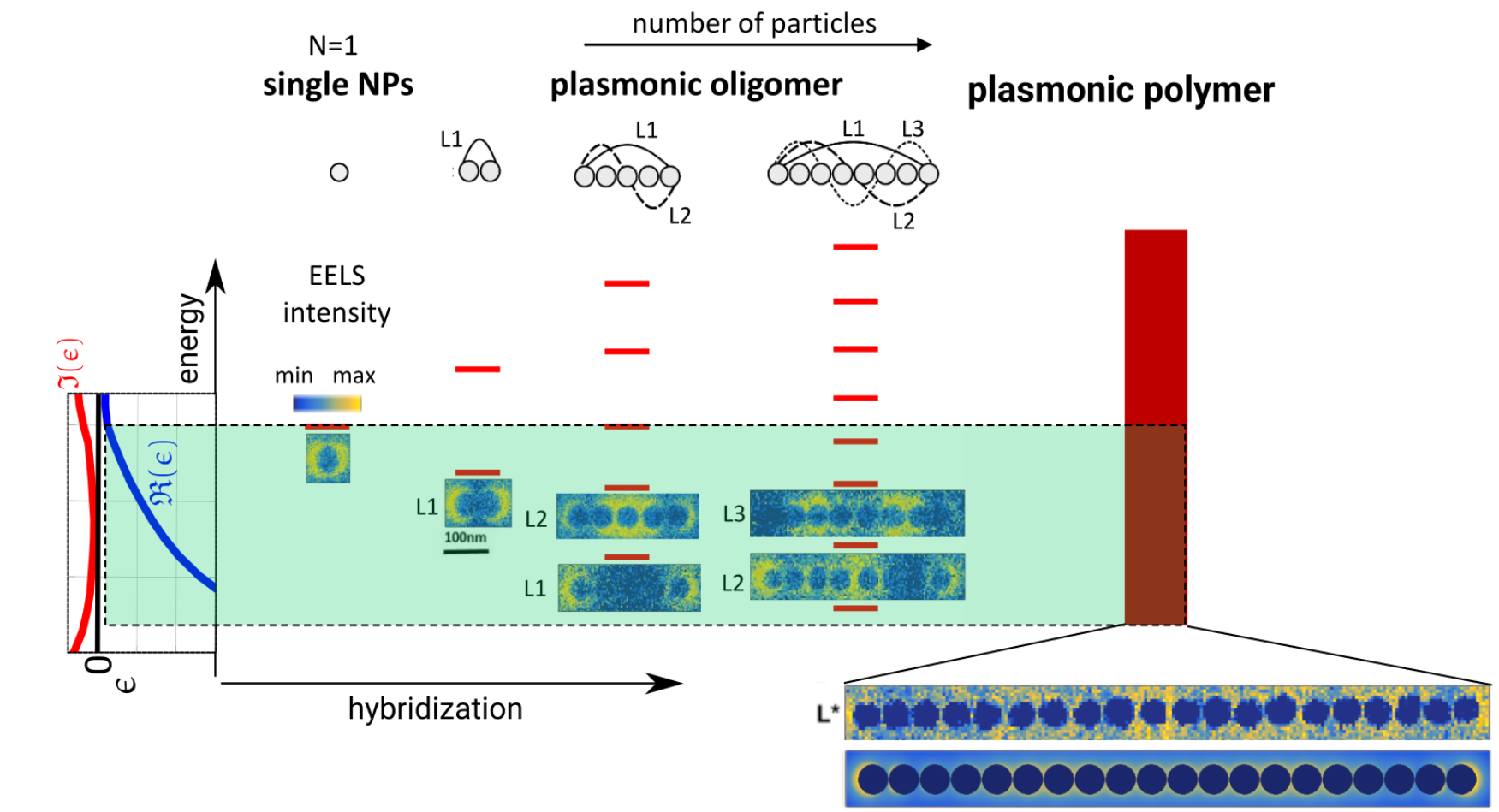

Figure 1. Figure 1: Plasmon Band Formation Principle. With increasing number of particles, the number of hybridized modes increases, eventually merging. The quasi-infinite chains show a delocalized surface plasmons and quasi-continuous plasmon bands. 

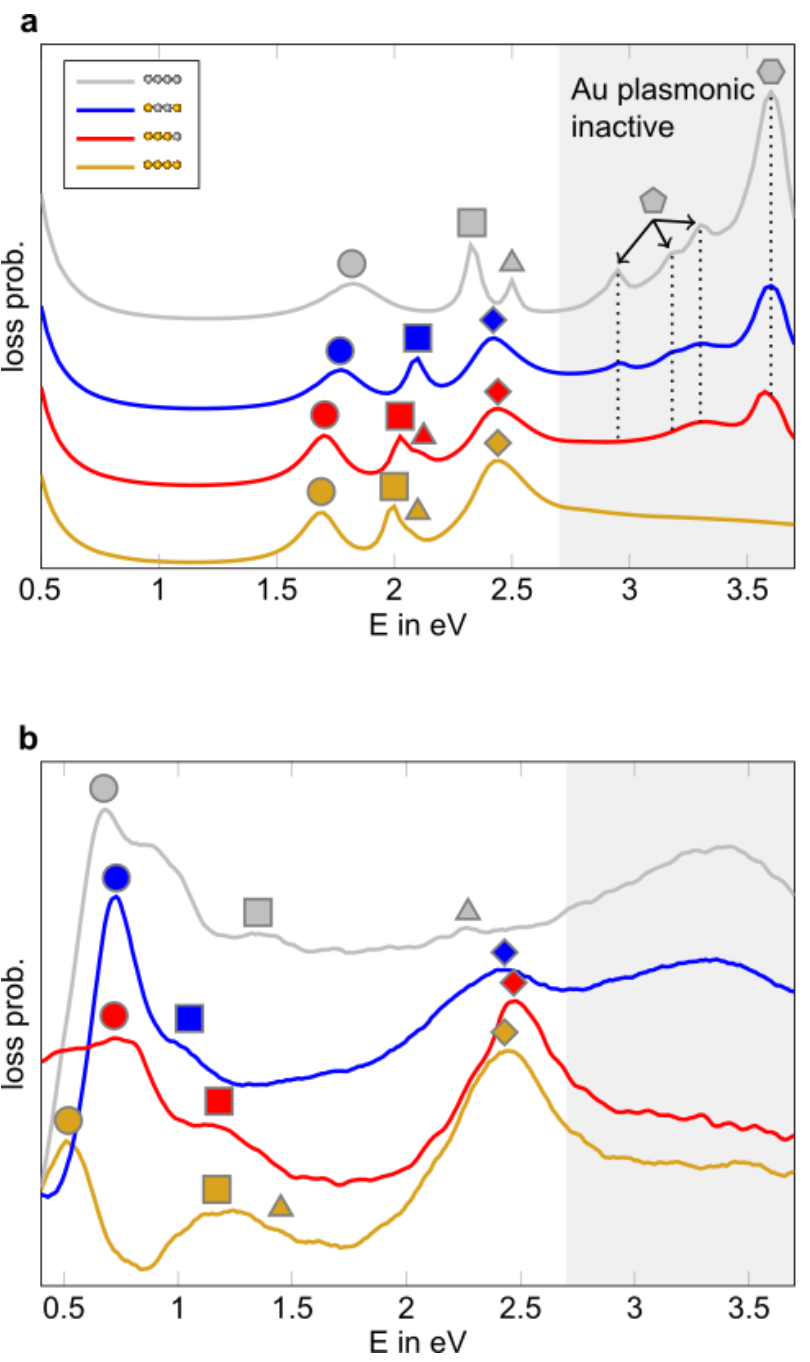

C
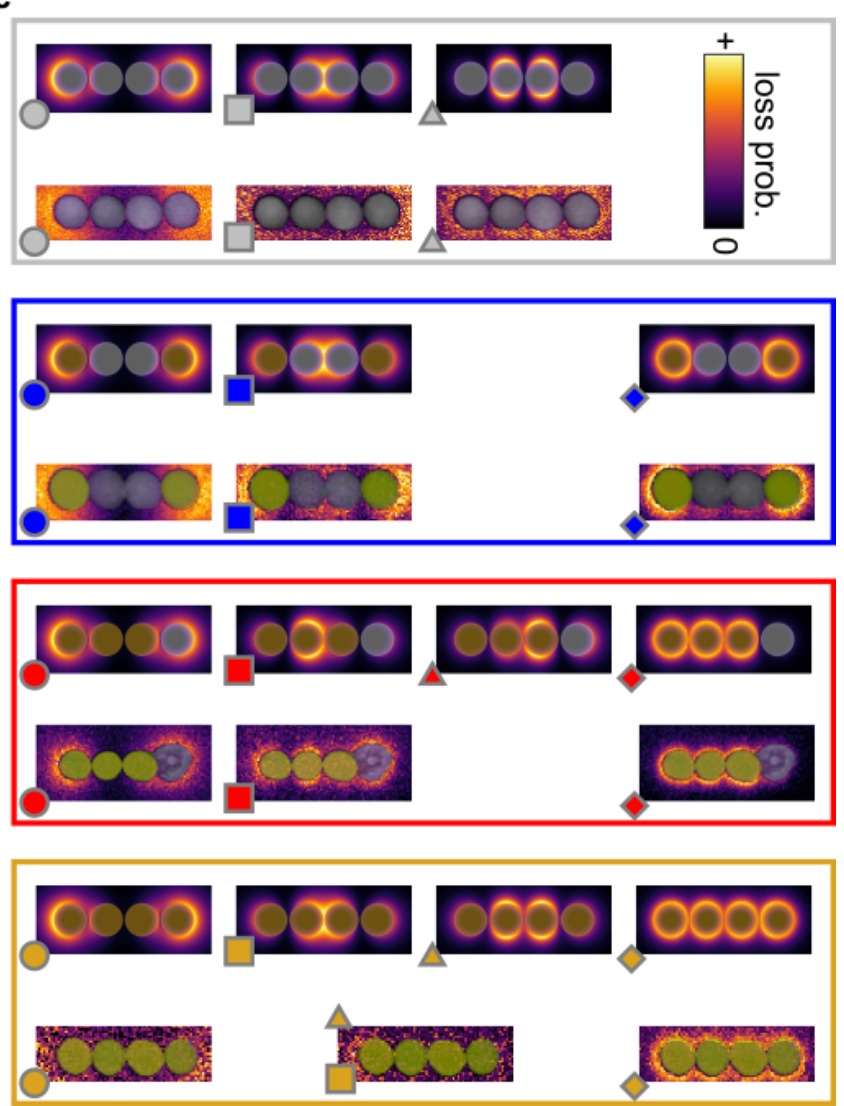
$\left(p_{*} p_{*} p_{*} p_{*}\right)_{1}^{-}$
$\left(p_{*} p_{*} p_{*} p_{*}\right)_{2}^{-}$
$\mathbf{\Delta}\left(\mathrm{p}_{*} \mathrm{~d}_{*} \mathrm{~d}_{*} \mathrm{p}_{*}\right)_{1}^{-}$
non-hybridized Au-modes
Ag bulk-mode
non-hybridized Ag-modes

Figure 2. Figure 2: Simulated a) and experimental b) spectra for hybridization in chains of four NPs with varying Ag-Au content [3]. The corresponding EELS maps are shown in c). The modes are denoted using angular momentum classification known from Mie Theory $(1=0: s, 1=1: p, \ldots)$. Modes indicated by circles, squares and triangles correspond to different px coupled modes. Higher order modes which couple weakly are denoted by diamonds (hexagons) in case of gold (silver). The silver-bulk mode is indicated by a hexagon. Plasmonic excitations of gold are suppressed in the gray shaded area due to its dielectric function.

\section{References}

[1] Nelayah et al., Nat. Phys. 2007, 3 (5), 1745-2481.

[2] Mayer et al., Nano Lett. 2019, 19 (6), 3854-3862.

[3] (Schletz, Schultz) et al., Adv. Optical Mater. 2021, 2001983. 